\title{
Review
}

\section{The New Era of Staging as a Key for an Appropriate Treatment for Esophageal Cancer}

\author{
Akira Tangoku, MD, PhD, Yota Yamamoto, MD, Yoshihito Furukita, MD, \\ Masakazu Goto, MD, and Masami Morimoto, MD
}

\begin{abstract}
Fluorodeoxyglucose-positron emission tomography (FDG-PET) and computed tomography (CT) have become the gold standard for staging of esophageal cancer by detecting distant metastases, but metastatic lymph nodes are often difficult to diagnose from the size and standardized uptake value (SUV). If we compare the diagnostic performance of endoscopic ultrasonography (EUS), CT, and FDG-PET in staging of esophageal cancer, EUS is the most sensitive method to identify the detection of regional lymph node metastases, whereas CT and FDG-PET are more specific tests. Combination study with CT, EUS and PETCT cannot make a precise diagnosis after neoadjuvant therapy (NAT). A precise staging might be determined by the fine needle aspiration biopsy (FNAB) under EUS and US screening in the neck and the abdomen even after NAT. Indication of endoscopic mucosal resection (EMR) and endoscopic submucosal dissection (ESD) for superficial cancer is sensitive because of difficulty in T1b cancer diagnosis. Detailed examination about vessel invasion and the possibility of residual tumor with dissected specimen will offer an appropriate additional therapy. New strategy like sentinel lymph node (SLN) navigation could supply more information about lymphatic routes and metastatic nodes. SLN navigation with ESD might become a new less invasive strategy for superficial esophageal cancer.
\end{abstract}

Keywords: esophageal cancer, staging, TNM, sentinel lymph node biopsy

\section{Introduction}

The esophagus is a tubular organ which exists in the posterior mediastinum. The lymphatic network is developed and widely spread, lymph node (LN) metastases are very common even in the case of superficial cancer not only in the mediastinum but also in the neck and the abdomen. ${ }^{1-5)}$ Therefore, the prognosis of thoracic esophageal

Department of Thoracic, Endocrine Surgery and Oncology, Institute of Health Bioscience, Tokushima University Graduate School, Kuramoto, Tokushima, Japan

Received: February 13, 2012; Accepted: March 21, 2012 Corresponding author: Akira Tangoku, MD, PhD. Department of Thoracic, Endocrine Surgery and Oncology, Institute of Health Bioscience, Tokushima University Graduate School, 3-18-15, Kuramoto, Tokushima 770-8505, Japan

Email: tangoku@clin.med.tokushima-u.ac.jp

(C)2012 The Editorial Committee of Annals of Thoracic and

Cardiovascular Surgery. All rights reserved. cancer has been improved since the prevalence of the three-field LN dissection. ${ }^{6,7)}$ The plenty number of stocked data from the patients have proved the accurate percentage of LN metastases and prognosis after surgery, according to the tumor extent. ${ }^{2-5,8)}$ Esophageal squamous cell carcinoma (ESSC) is known to be sensitive to chemo-radiotherapy (CRT). Recent improvements of clinical results in CRT also added the choice for treatment. These data realized a tailor-made option in therapeutic strategy for each patient. However, an accurate diagnosis of tumor invasion and metastasis is key for the choice of an appropriate treatment. Development of the endoscope, multi detector computed tomography (MDCT) scan, magnetic resonance imaging system (MRI), and 18F-fluoro-2-deoxy-D-glucose positron emission tomography $\left({ }^{18} \mathrm{~F}-\mathrm{FDG}-\mathrm{PET}\right)$ have obtained the accurate diagnosis about tumor extent of esophageal cancer. This review shows recent advances concerning staging and therapeutic modality for esophageal cancer. 


\section{TNM Staging}

Based on the tumor staging, malignant potential, and patient's physical status, the choice of strategies are informed to patients with the basis and the process of decision making. Then the appropriate treatment is selected (Fig. 1) ${ }^{8)}$ Stages of esophageal cancer are represented by $\mathrm{T}$ (primary tumor), $\mathrm{N}$ (regional lymph nodes), and $\mathrm{M}$ (distant metastases). TNM staging were first introduced by the UICC (International Union Against Cancer) in 1968. In 1977, the first edition of the AJCC (American Joint Committee on Cancer) Manual for Staging Cancer introduced its TNM classifications and stage groupings for esophageal cancer. In 1992, the fourth edition did not change in esophageal cancer staging. In 1997, the fifth edition introduced a sub classification of staging: M1 became M1a (cervical lymph node metastasis for cancers of the upper thoracic esophagus and celiac lymph node metastasis for cancer of the lower thoracic esophagus) and M1b (all other distant metastasis), and the subclassification of stage IV into IVA and IVB. The sixth edition, published in 2002, was unchanged from the fifth edition.")

In the seventh edition, released in 2010, $\mathrm{T}$ is classified as Tis for high-grade dysplasia, T1 for cancer that invades the lamina propria, muscularis mucosae, or submucosa; $\mathrm{T} 2$ for cancer that invades the muscularis propria; $\mathrm{T} 3$ for cancer that invades the adventitia, T4a for resectable cancer that invades adjacent structures such as pleura, pericardium, or diaphragm, and T4b for unresectable cancer that invades other adjacent structures, such as aorta, vertebral body, or trachea. $\mathrm{N}$ is classified as $\mathrm{N} 0$, no regional $\mathrm{LN}$ metastasis; $\mathrm{N} 1$, regional LN metastases involving 1 to 2 nodes; N2, regional LN metastases involving 3 to 6 nodes; and N3, regional LN metastases involving 7 or more nodes. $\mathrm{M}$ is classified as $\mathrm{M} 0$, no distant metastasis; and M1, distant metastasis., $\left.{ }^{9} 10\right)$

The AJCC addressed the importance of identifying nonanatomic classificationsthat depend on histopathologic type, grade, and tumor location, concerning recent clinical data and cancer biology. Difference in survival between adenocarcinoma and squamous cell carcinoma is managed by separate stage groupings for stages I and II. Histologic grade is associated with survival for early-stage cancers. For adenocarcinoma, G1 and G2 (well and moderately differentiated) distinguished from G3 (poorly differentiated) for stage I and stage IIA cancers. For squamous cell carcinoma, G1 is distinguished from G2 and G3 for stage I and II cancers. Tumor location (upper and middle thoracic versus lower thoracic) is an important factor for grouping T2-

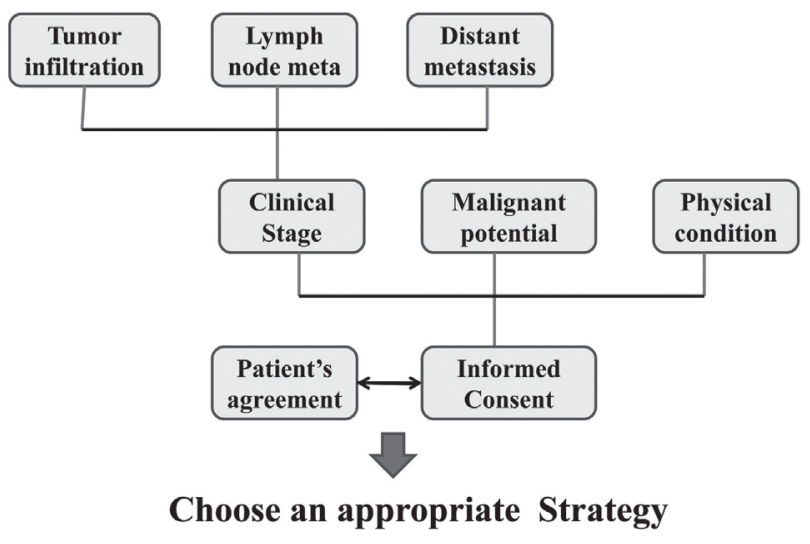

Fig. 1 Decision making tree for esophageal cancer therapy.

A critical component of choosing an appropriate management strategy is an accurate assessment of tumor extent, which is decided from the experimental data about tumor infiltration, number and location of the lymph node metastasis, existence of distant metastasis, and patient's physical condition. (The Japan Esophageal Society. Guidelines for diagnosis and treatment for esophageal cancer: The 2nd Edition, Revised Version, Kanahara, Tokyo, 2007). ${ }^{8)}$

3NOM0 squamous cell cancers. For T1NOM0 and T2NOM0 adenocarcinoma, subgrouping is made by histologic grade: G1 and G2 versus G3. Stage groupings for T1N0M0 squamous cell carcinoma, subgrouping is made by histologic grade: G1 versus G2 and G3. For T2NOM0 and T3N0M0 squamous cell carcinoma, stage grouping is made by histologic grade and location. The four combinations range from G1 lower thoracic squamous cell carcinoma (stage IB), to G2-G4 upper and middle thoracic squamous cell carcinomas (Stage IIB), which have the worst grades. G2-G4 lower thoracic squamous cell carcinomas and G1 upper and middle thoracic squamous cell carcinomas are grouped together (stage IIA), with intermediate survival. Stage 0, III, and IV adenocarcinoma and squamous cell carcinoma are identically stage grouped. However, these new stage classifications are not included in UICC TNM 7th edition yet. ${ }^{9,10)}$

The current TNM staging system of the Japan Esophageal Society TNM classification (The 10th Edition) defines tumor extent classified as follows: TX (primary tumor cannot be evaluated), T0 (no evidence of primary tumor), Tis (carcinoma in situ, epithelial cancer), T1 (infiltration of lamina propria or tunica submucosa), T2 (infiltration of tunica muscularis propria), T3 (infiltration to adventitia), and T4 (infiltration to mediastinal structures). Regional lymph nodes are classified as follows: $\mathrm{Nx}$, regional $\mathrm{LN}$ cannot be evaluated; N0, no regional LN; N1, regional (group 1) LN metastases; N2, metastases to group $2 \mathrm{LN}$; N3, metastases to group $3 \mathrm{LN} ; \mathrm{N} 4$, metastases to distant $\mathrm{LN}$ far from group 
Table 1 Comparison between UICC TNM classification 7th edition ${ }^{10)}$ and Japan Esophageal Society TNM classification 10th edition ${ }^{11)}$

\begin{tabular}{|c|c|c|c|}
\hline \multicolumn{2}{|r|}{ UICC TNM classification } & \multicolumn{2}{|r|}{ Japan Esophageal Society TNM classification } \\
\hline \multicolumn{4}{|c|}{ Primary Tumor $(\mathrm{T})$} \\
\hline TX & Primary tumor cannot be assessed & TX & primary tumor cannot be evaluated \\
\hline T0 & No evidence of primary tumor & T0 & no evidence of primary tumor \\
\hline Tis & High-grade dysplasia* & Tis & carcinoma in situ; epithelial cancer \\
\hline $\mathrm{T} 1$ & $\begin{array}{l}\text { Tumor invades lamina propria, muscularis mucosae, } \\
\text { or submucosa }\end{array}$ & $\mathrm{T} 1$ & infiltration of lamina propria or tunica submucosa \\
\hline T1a & Tumor invades lamina propria or muscularis mucosa & T1a & 1ainfiltration of lamina propria or muscularis mucosae \\
\hline $\mathrm{T} 1 \mathrm{~b}$ & Tumor invades submucosa & $\mathrm{T} 1 \mathrm{~b}$ & Tumor invades submucosa \\
\hline $\mathrm{T} 2$ & Tumor invades muscularis propria & $\mathrm{T} 2$ & Tumor invades muscularis propria \\
\hline $\mathrm{T} 3$ & Tumor invades adventitia & $\mathrm{T} 3$ & Tumor invades adventitia \\
\hline $\mathrm{T} 4$ & Tumor invades adjacent structures & $\mathrm{T} 4$ & Tumor invades mediastinal adjacent structures \\
\hline $\mathrm{T} 4 \mathrm{a}$ & $\begin{array}{l}\text { Resectable tumor invading pleura, pericardium, or } \\
\text { diaphragm }\end{array}$ & & \\
\hline $\mathrm{T} 4 \mathrm{~b}$ & $\begin{array}{l}\text { Unresectable tumor invading other adjacent structures, } \\
\text { such as aorta, vertebral body, trachea, etc. }\end{array}$ & & \\
\hline \multicolumn{4}{|c|}{ Regional Lymph Nodes $(\mathrm{N})^{* *}$} \\
\hline NX & Regional lymph nodes cannot be assessed & NX & Regional lymph nodes cannot be assessed \\
\hline NO & No regional lymph node metastasis & No & no regional lymph nodes \\
\hline N1 & Regional lymph node metastases involving 1 to 2 nodes & N1 & regional (group 1) ${ }^{\#}$ lymph node metastases \\
\hline $\mathrm{N} 2$ & Regional lymph node metastases involving 3 to 6 nodes & $\mathrm{N} 2$ & regional (group 2) " lymph node metastases \\
\hline \multirow[t]{2}{*}{ N3 } & Regional lymph node metastases involving 7 or & N3 & regional (group 3) ${ }^{\#}$ lymph node metastases \\
\hline & more nodes & N4 & distant (far from group 3) lymph node metastases \\
\hline \multicolumn{4}{|c|}{ Distant Metastasis $(\mathrm{M})$} \\
\hline MX & Distant metastasis cannot be assessed & MX & Distant metastasis cannot be assessed \\
\hline M0 & No distant metastasi & M0 & No distant metastasi \\
\hline M1 & Distant metastasis & M1 & Distant metastasis \\
\hline
\end{tabular}

* Includes all noninvasive neoplastic epithelium that was previously called carcinoma in situ. Cancers stated to be noninvasive or in situ are classified as Tis.

** Number must be recorded for total number of regional nodes sampled and total number of reported nodes with metastases.

\# Lymph node group is defined by main location of the tumor.

3. The group of $\mathrm{LN}$ is defined by location of the primary tumor. Distant metastases are classified as follows: Mx, distant metastasis cannot be evaluated; M0, no distant metastases; M1, distant metastasis is present. TNM combinations correspond to one of the following stages: stage $0, \mathrm{~T} 0$, T1a and N0; stage I, T1b N0 M0 and T0/1a, N1, M0; stage II, T2/3 N0 M0, T1b/2 N1 M0, T0/1a N2 M0; stage III, T4 N0 M0, T3 N1 M0, T2/3 N2 M0, T0-3 N3 M0; Stage IVa, T4 N1-3 M0, T1-4 N4 M0; Stage IVb, T0-4 N0-4 M1. ${ }^{11)}$ A comparison of TNM classifications: UICC version 7 with the Japan Esophageal Society 10th edition is shown in Table.

\section{Diagnosis for Tumor Infiltration of Superficial Cancer}

Superficial esophageal cancers are categorized as an in situ lesion (Tis) and T1 tumor. T1 are split into T1a and T1b subcategories, depending on the depth of invasion. T1a (M1 or SSM, intraepithelial cancer; M2 or LPM, cancer with invasion into the lamina propria mucosae; M3 or $\mathrm{DMM}$, cancer reaching the muscularis mucosae), $\mathrm{T} 1 \mathrm{~b}$
(SM1, cancer with invasion into one third of the submucosa; SM2, cancer with invasion into the middle third of the submucosa; SM3, cancer with invasion into the lower third of submucosa). ${ }^{8,11)}$ As the result of analyses of superficial ESCC in Japan, the incidence of lymph node metastasis in M1, M2, and M3 was $0 \%, 3.3 \%$, and $12.2 \%$. In submucosal cancer, nodal metastasis was found in $26.5 \%$ of SM1, 35.8\% of SM2, and 45.9\% of SM3 (Fig. 2)., ${ }^{4}$ )

Endoscopic treatments, such as endoscopic mucosal resection (EMR) and endoscopic submucosal dissection (ESD), have recently become possible choices for intraepithelial neoplasia. Based on the incidence of LN metastasis, the guideline recommends an absolute indication of EMR or ESD for an M1 or M2 tumor and a relative indication for an M3 or SM1 tumor. Almost half of Japanese patients with superficial ESCC underwent EMR or ESD, and the remaining half underwent surgical treatment. ${ }^{12)}$ However, these techniques are appropriate for patients with no lymph nodes metastasis, whoare expected to be poor candidates for invasive esophageal surgery.

A critical component for choosing an appropriate 


\section{Tumor extent and lymph node metastatic rate of Clinical Stage I esophageal cancer}

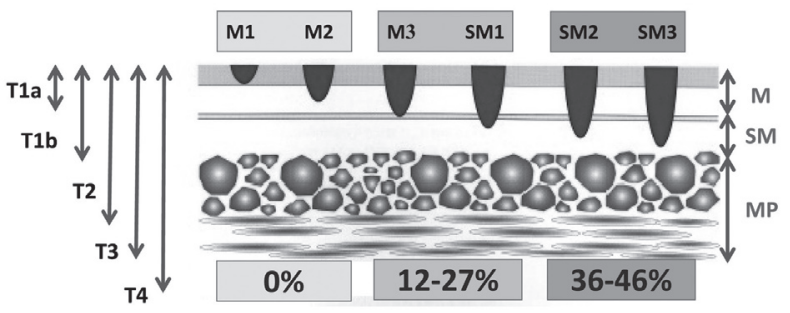

Fig. 2 Tumor infiltration and lymph node metastases of T1 cancer. Early esophageal cancers are in situ lesion (Tis) or T1 tumors. T1 are split into Tla and Tlb subcategories depending on the depth of invasion. T1a ( $\mathrm{m} 1$ or SSM, intraepithelial cancer; $\mathrm{m} 2$ or LPM, cancer with invasion into the lamina propria mucosae; $\mathrm{m} 3$ or DMM, cancer reaching to the muscularis mucosae), T1b (sm1, cancer with invasion into one third of the submucosa; sm2, cancer with invasion into the middle third of the submucosa; sm3, cancer with invasion into the lower third of submucosa). The incidence of lymph node metastasis in M1, M2, and M3 was $0 \%, 3.3 \%$, and $12.2 \%$, respectively. In submucosal cancer, nodal metastasis was found in $26.5 \%$ of SM1, 35.8\% of SM2, and $45.9 \%$ of SM3. ${ }^{4,8)}$

management strategy for a superficial cancer is an accurate assessment of the tumor extent, using narrow band imaging (NBI) endoscopy and high frequency endoscopic ultrasonography (EUS). The NBI endoscope became the standard examination for the early detection of superficial esophageal cancer for identifying the intrapapillary capillary loop pattern. ${ }^{13,14)}$ However, the views of Japanese and Western pathologists have differed significantly. Before the term "intraepithelial neoplasia" was introduced, severe dysplasia as diagnosed by Western pathologists was the same as carcinoma in situ or noninvasive carcinoma as diagnosed by Japanese pathologists. This problem has been solved by the introduction of the Vienna classification; however, there are still some issues that need to be resolved. One of them is the presence of basal layer type carcinoma in situ, which is often underdiagnosed as low grade intraepithelial neoplasia by Western pathologists. ${ }^{14)}$

\section{Modality for Tumor Staging}

Tumor staging is decided with several imaging modalities. After diagnosis with endoscopic biopsy and pathology, tumor extent is examined with EUS, upper gastrointestinal series (fluorography), CT scan, MRI, FDG-PET, bone scintigraphy and US. The guideline shows that the diagnosis should be made by the use of endoscopy and fluorography in patients with symptoms. Endoscopic examination is recommended for patients without symptoms.
Endoscopy that includes iodine staining, and fluorography, EUS, CT scan, and MRI are recommended for explanation of tumor extent. ${ }^{8)}$ Metastases to other organs, including LNs, are diagnosed using a CT scan, MRI, EUS, bone scintigraphy, and FDG-PET/CT. Cervical and abdominal US also provide important information for staging diagnosis. Generally, Tumor length and width is measured by the use of fluorography and CT scan andinvasion to neighboring organs and metastasis are diagnosed with CT scan and MRI. Distant organ metastases can be well figured out by FDG-PET/CT., ${ }^{8,11)}$

EUS is a useful procedure for distinguishing T1 lesion from deeper infiltration. Meta-analysis of 19 international studies showed the sensitivity and specificity of the EUS for T1a staging were 0.85 and $0.87,0.86$ and 0.86 for T1b. ${ }^{15)}$ Especially in T staging, EUS could play an important role in the choice of candidates for endoscopic and surgical treatment. Locoregional invasion in esophageal cancer can be predicted by PET/CT. The SUVmax of the primary tumor helps to identify patients with $\mathrm{T} 1$ tumors. Positive regional node SUVmax can help evaluate the severity of nodal involvement. However, the sensitivity, specificity, and accuracy of PET/CT were only 57.1\%, $83.3 \%$, and $71.1 \%$, and even lower for detecting non regional lymph node metastasis. ${ }^{16)}$ It is not recommended to use FDG-PET or PET/CT alone as a diagnostic tool to determine clinical target volume (CTV) if pathologically involved lymphatic regions are to be included in the CTV in the treatment protocol. The accuracy of FDG-PET/ CT must be further improved in order to better detect positive nodes and improve the definition of the CTV. ${ }^{17}$

\section{Modality for Lymph Node Metastases}

Mediastinal LNs, especially those neighboring the trachea and main bronchus, are often large and round. Those are difficult to distinguish from metastasis, in size and shape. Okada et al. retrospectively reviewed regional LNs of esophageal cancer on contrast enhanced CT (CECT) images by two blinded evaluators on the basis of the following cutoff sizes: 7 $\mathrm{mm}$ for all regional LNs (Protocol A), $10 \mathrm{~mm}$ for paratracheal LNs (Protocol B), and $7 \mathrm{~mm}$ for others. In addition, the maximum standardized uptake value (SUVmax) on PET/CT was evaluated for positive uptake by LNs. The sensitivity, specificity, accuracy, positive, and negative predictive values of PET/CT were $60.0 \%, 99.5 \%, 94.8 \%, 93.8 \%$, and $94.8 \%$, whereas those of CECT were $60.0 \%, 95.1 \%, 91.0 \%, 62.5 \%$, and $94.6 \%$ (Protocol A) and 56.0\%, 97.3\%, 92.4\%, 73.7\%, and $94.2 \%$ (Protocol B). Integrated PET/CT improves 
the PPV of regional LNs when compared with CECT. The smallest LN metastasis detectable by PET/CT was $6 \mathrm{~mm}{ }^{18)}$ Meta-analysis for comparing EUS, CT and FDG-PET showed pooled sensitivities of EUS, CT, and FDG-PET for regional $\mathrm{LN}$ metastases were $0.80,0.50$, and 0.57 , and specificities were $0.70,0.83$, and 0.85 . For detection of celiac LN metastases by EUS, sensitivity and specificity were 0.85 and 0.96. For abdominal LN metastases by CT, these values were 0.42 and 0.93 . For distant metastases, sensitivity and specificity were 0.71 and 0.93 for FDG-PET and 0.52 and 0.91 for CT. Diagnostic performance of FDG-PET for distant metastases was significantly higher than that of CT, which was not significantly affected by study and patient characteristics. ${ }^{19)}$

LN metastasis, beside the esophagus, is also diagnosed with EUS. The overall accuracy of EUS for T staging was $72 \%$, and it was the only method for delineating the layers of the esophageal wall. The sensitivities for $\mathrm{N}$ staging were $42 \%$ for EUS, $49 \%$ for PET, and $35 \%$ for CT, and their specificities were 91,87 , and 93\%. The accuracy for $\mathrm{N}$ staging was $66 \%$ for EUS, $68 \%$ for PET, and $63 \%$ for $\mathrm{CT}$, and it did not differ significantly across the three tests. The author concluded that preoperative EUS for the loco-regional staging of esophageal cancer provides excellent $\mathrm{T}$ staging accuracy and similar accuracy for $\mathrm{N}$ staging, compared with PET and CT. ${ }^{20)}$

In the comparison of the diagnostic performance of EUS, CT, and FDG-PET in staging of esophageal cancer, EUS is the most sensitive in identifying the detection of regional LN metastases, whereas CT and FDG-PET are more specific tests. ${ }^{21)}$

Patients proven by endoscopy and biopsy with no evidence of distant metastatic disease on CT and FDG-PET were referred for EUS for locoregional staging. The results of $\mathrm{N}$ staging with CT, FDG-PET, and EUS were compared with surgical pathology or EUS-FNA cytology. Overall accuracy for $\mathrm{N}$ staging was $69 \%$ for CT, $56 \%$ for FDG-PET, and $81 \%$ for EUS. The combination of CT plus EUS appears to be accurate for locoregional staging in esophageal cancer. ${ }^{21)}$ EUS is also the most accurate technique for preoperative local-regional staging of esophageal carcinoma, once CT and/or the FDG-PET scan have excluded the presence of distant metastasis. EUS-guided fine needle aspiration (EUS-FNA) might help improve diagnostic accuracy in esophageal cancer LN staging, and the therapeutic decision might be derived from such a practice. ${ }^{21)}$ Omloo et al. emphasized an additional value of external US of the neck as a part of the routine diagnostic work-up in patients with esophageal cancer, even after normal CT and FDG-PET scanning. ${ }^{22)}$

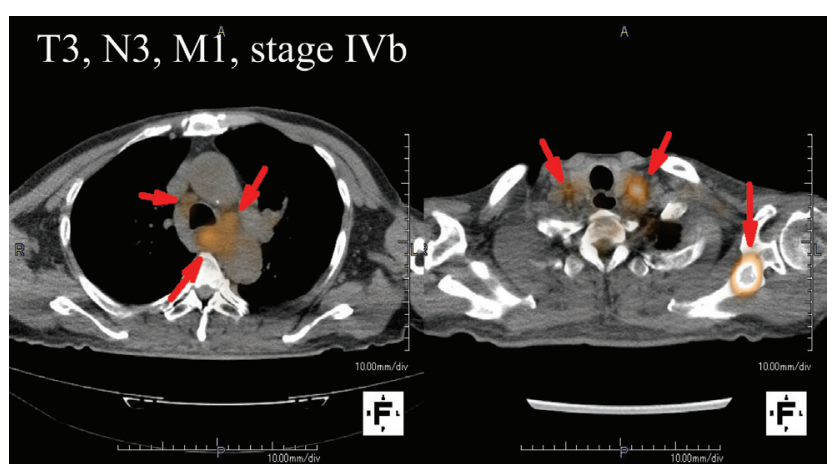

Fig. 3 Distant metastases diagnosed by FDG-PET and CT scan. FDG-PET staging is more accurate than CT staging in preoperative staging of esophageal cancer. Combined use of these modalities can detect possible metastases, and CT can confirm or exclude their presence and precisely determine the locations. In this case, PET-CT identified LN and bone metastasis to scapula bone. There are many false positives and false negatives in PET diagnosis. Interpretation of the PET/CT results is optimized by an understanding of the diagnostic limitations and pitfalls that may be encountered, together with a knowledge of the natural history of esophageal cancer and staging and treatment options available.

\section{Modality for Distant Metastases}

For the evaluation of distant metastases, FDG-PET has probably a higher sensitivity than CT. Recent study showed FDG-PET staging was more accurate than CT in the preoperative staging of esophageal cancer. ${ }^{23)}$ The combined use of these modalities could, however, be of clinical value, with FDG-PET detecting possible metastases and CT confirming or excluding their presence and precisely determining the locations (Fig. 3).

As for bone metastasis, there is a meta-analysis comparing FDG-PET and bone scintigraphy in the detection of osseous metastases in patients with breast cancer. The study evaluated the diagnostic accuracy between two modality by systematic surveillance of MEDLINE, CINAHL, EBM Review databases. The authors concluded that FDG-PET does have a higher specificity (95\% vs. $79 \%$ ) and may serve as a confirmatory test than bone scintigraphy and used to monitor response to therapy. ${ }^{24)}$

\section{New Therapeutic Strategy for Stage II, III Esophageal Cancer}

Results of phase II study which examined the efficacy of CRT for Stage II, III

Esophageal cancer (Japan clinical oncology group: JCOG9906 study) ${ }^{25)}$ could not show superior survival benefit compared with previous surgical result and high toxicity especially in the late phase was shown to be a critical problem. 


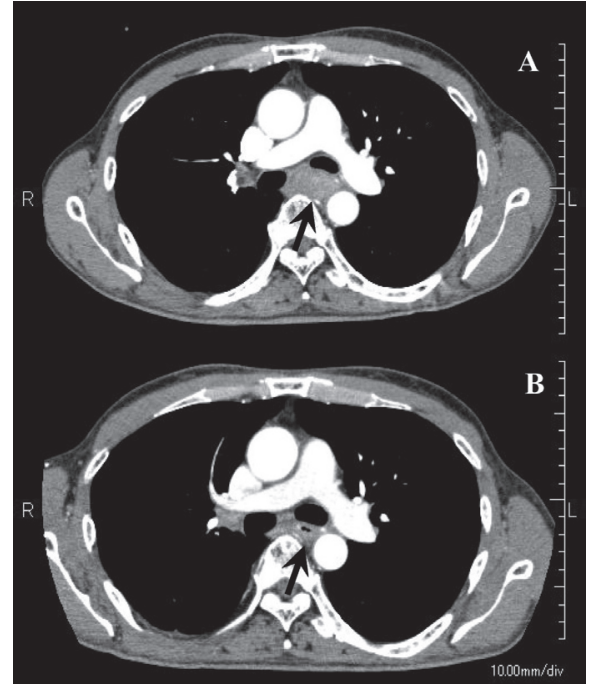

Fig. 4 Evaluation of primary chemotherapy with CT.

Invasion into a neighboring organ can be diagnosed with enhanced CT scan. Esophageal cancer (arrow) in the patient was suspected to have invaded the aorta and the left main bronchus (A). Tumor shrunken after the NAC with decetaxel, cisplatin, and 5-FU, and curative surgery has performed (B).

Many patients needed a salvage operation, which brought about serious mortality and morbidity. ${ }^{26)}$ The JCOG9204 study showed efficacy of adjuvant chemotherapy, with CDDP and 5FU increasing the five year survival rate (55\% vs. $45 \%) .{ }^{27)} \mathrm{A}$ randomized study comparing adjuvant and neo-adjuvant chemotherapy (NAC) (JCOG9907) showed that NAC increased compliance of the therapy $(85.4 \%$ vs. $75 \%$ ), and down staging was achieved in some patients by NAC. ${ }^{28,29)}$ The five-year overall survival rate was better in NAC group (55\% vs $43 \%, \mathrm{p}=0.04$ ); however, progression free survival did not reach the stopping boundary. ${ }^{28)}$

NAC with low dose of CDDP and 5-FU combined with weekly Docetaxel had obtained a better response rate and a more pathological complete response than the ordinary NAC with CDDP and 5FU (Fig. 4). ${ }^{30)}$ Recently, our data showed that patients with positive EGFR had a poor prognosis without chemotherapy; however, the prognosis had improved with NAC. EGFR is a predictive factor in the patient's prognosis and response to chemotherapy, and HER2 and HER3 also show similar behaviors. NAC with taxane was recommended for ESCC patients with positive for HER family members. ${ }^{31)}$ These biomarkers will be candidates for the predictor of prognosis (recurrent risk) and choosing the therapeutic options. Molecular therapy targeting these biomarkers might improve the prognosis of esophageal cancer. ${ }^{32)}$

These data show that neoadjuvant therapy (NAT)becomes a common choice for treatment of ECSS in Japan, therefore, an accurate estimation of NAT is essential to

\section{Strategies for esophageal cancer}

Clinical stage is diagnosed based on Endoscopy,

EUS, Fluorography, CT, MRI, and FDG/PET-CT

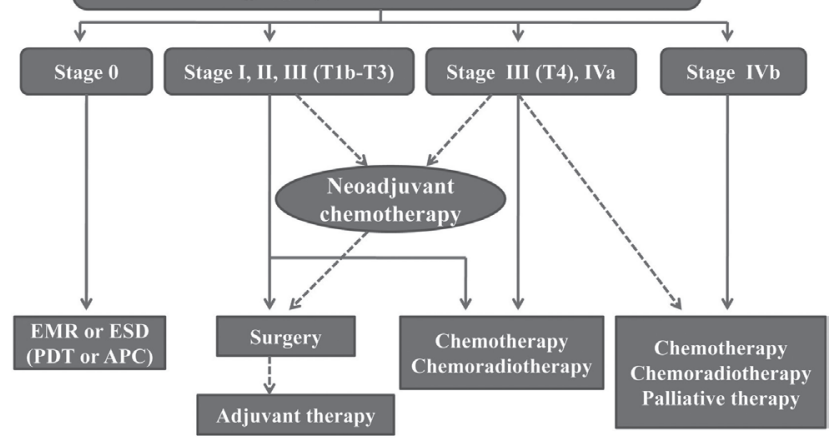

Fig. 5 Strategies for esophageal cancer.

Therapeutic strategy is chosen according to tumor stage. For stage II and III cancers, NAC becomes a common choice for treatment of ESCC in Japan. An accurate estimation of NAT is essential for deciding the following therapy, but re-estimation of the advanced tumor is still controversial. (The Japan Esophageal Society: Guidelines for diagnosis and treatment for esophageal cancer. (The 2nd Edition, Reviced Version), Kanahara, Tokyo, 2007) ${ }^{8)}$

decide the following therapy (Fig 5) ${ }^{8}{ }^{8}$ But re-estimation of the advanced tumor is still controversial. Especially, diagnosis of invasion to neighboring organs is difficult after CRT even with EUS, enhanced CT scan and MRI.

\section{Evaluation of Primary Therapy and Restaging}

FDG-PET is a good modality for predicting the efficacy of NAC and neoadjuvant chemoradiotherapy (NACRT) for advanced esophageal cancer. ${ }^{33,34)}$ An SUVmax reduction of more than $70 \%$ was classified as an FDG-PET responder and showed better prognosis, however, the response for the primary tumor (PT) or LN on CT evaluation was not a significant prognostic predictor. They concluded that FDGPET was superior to CT for evaluating the CRT response, from the viewpoint of survival analysis. ${ }^{33)}$ However, the response should be evaluated for both LN and PT because of their different behaviors during primary therapy. FDGPET/CT has limited utility in T staging and relatively limited utility in detection of dissemination to locoregional lymph nodes. ${ }^{34)}$ Interpretation of FDG-PET /CT results is optimized by an understanding of the diagnostic limitations and pitfalls that may be encountered together with knowledge of the natural history of esophageal cancer and the staging and treatment options available. ${ }^{35)}$

A recent systemic review concluded that PET was recommended to improve the accuracy of $\mathrm{M}$ staging for the staging work-up of patients who were potential candidates for 
curative therapy; however, no recommendation was made for or against the use of PET for the assessment of treatment response and evaluation of suspected recurrence. ${ }^{36)}$

A $50 \%$ or more reduction of tumor thickness by EUS post chemotherapy continues to be the best measure for tumor downstaging survival, while FDG-PET/CT may be more accurate than EUS-FNA and CT scan for predicting nodal status and complete responders after NAT. The role of EUS in restaging following NAT remains controversial, with recent studies showing that FDG-PET/CT may be more accurate than EUS-FNA and CT scan for predicting nodal status and complete responders after NAT. Potential methylation analysis, digital image analysis, and fluorescence in-situ hybridization on EUS-FNA samples might increase the yield and prove to be better than routine cytology ${ }^{37)}$ It is also hard to recognize dissectability of the fibrotic tissue including tumor after definitive CRT. Dynamic study with MDCT or MRI, or EUS elastography might help to diagnosis for dissectability. ${ }^{38)}$

\section{New Strategy for Superficial Esophageal Can- cer Diagnosis}

An accuracy of tumor depth for $\mathrm{m} 1 \mathrm{~m} 2$ cancer was $95 \%$, but it was $74 \%$ for $\mathrm{m} 3 \mathrm{sm} 1$, and $79 \%$ even with NBI scope. It is not satisfied because $20-30 \%$ of submucosal esophageal cancers which involves $\mathrm{LN}$ metastases are underestimated. ${ }^{39)}$

EMR and ESD have been developed in Japan. Pathological mapping and decision making for further treatment are feasible with those enhanced biopsy specimen. Phase II study of CRT for stage I (cT1bN0) esophageal cancer (JCOG9708) proved efficacy and safety of CRT from the viewpoint of organ preservation. ${ }^{40)}$ Additional studies are necessary to further elucidate this multimodality approach. ${ }^{41)}$ There is currently an ongoing phase II study evaluating the efficacy and the safety of combined treatment of EMR and CRT for clinical stage I esophageal cancer (JCOG0508). In this study, EMR was made for T1b (sm 1, 2 suspected, $<5 \mathrm{~cm},<3 / 4,<2$ accessory lesion) NOM0 patients. An additional CRT; two cycles of 5-FU/ cisplatin, with 41.4 Gy of radiation for negative resection margin or 50.4 Gy with boost on the primary site for positive margin is added if residual tumor is suspected after detailed pathological examination. The data from this trial will be expected to provide a non-surgical treatment option to clinical stage I (T1b) esophageal cancer patients. ${ }^{42)}$

\section{Another Strategy for Measuring Tumor Extent of Superficial Esophageal Cancer}

The sentinel lymph node (SLN) concept has been developed by researchers in several different cancer centers and has become a widely accepted element in the routine surgical management of breast cancer. ${ }^{43)}$ It has been reported that $\mathrm{pNO}$ breast cancer patients diagnosed by SLN biopsy (SLNB) showed superior survival to pN0 patients diagnosed by axillary dissection. It is easily acceptable because SLNs are examined more carefully in thin sliced spacemen compared with dissected lymph nodes samples are examined in one sliced specimen. ${ }^{44)}$ Thus, SLNB provides strong evidence for an improvement in tumor staging on the basis of SLNB. At the moment, the available data does not justify reduced the extent of lymphadenectomy, but most recent result of phase III trial comparing SLNB alone and axillary lymph node dissection after SLNB for breast cancer patients with less than 2 node involvement showed no differences in local control and survival in breast cancer. ${ }^{45}$

Introduction of SLN navigation to gastrointestinal (GI) tract is controversial. SLNB using available techniques have suggested that the lymphatic drainage of GI tract is much more complicated than other sites, skip metastasis being rather frequent because of an aberrant lymphatic drainage outside of the basin exist. Recent studies have shown favorable results for identification of SLN in esophageal cancer. ${ }^{46)}$ Uenosono et al. performed SLN navigation surgery for T1, T2 and T3 patients with esophageal cancer after (99m) Tc-Tin colloid endoscopical injection into the esophageal wall around the tumor 1 day before surgery. They concluded SLN mapping could be applied to patients with cT1 and cN0 esophageal cancer. ${ }^{47)}$ The SLN concept might enable surgeons to perform less invasive surgery, with a reduction in the number of lymphadenectomies performed. However, SLN navigation with radioisotope and blue dye are the intra-operative technique. Radio-scintigraphy cannot supply preoperative detailed anatomical information, especially SLN neighboring the tumor cannot detect because of the shinethrough phenomenon. Tumor-occupied lymph nodes could not be detected preoperatively, and they were also missed during the operation. ${ }^{47)}$ We developed a new strategy of SLN mapping with CT scan. Lymph flows from the tumor was visualized on a $3 \mathrm{D}$ CT image, with the surrounding anatomy during the preoperative, routine $\mathrm{CT}$ examination for tumor infiltration and metastasis. ${ }^{48,49)}$

CT lymphography (CTLG) with endoscopic mucosal injection of iopamidol was applicable for SLN navigation of 
3D CTLG ( MIP, SSD images )

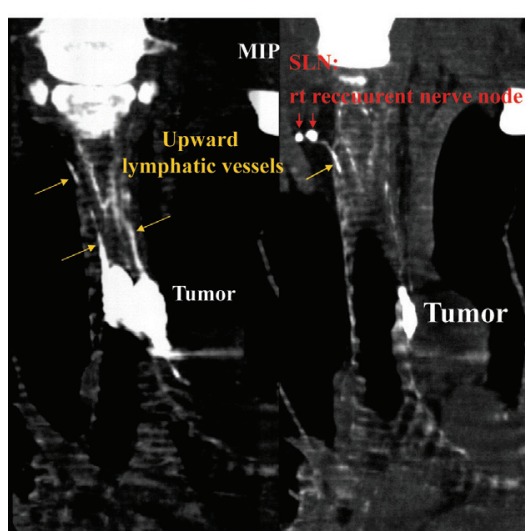

\section{CTLG ( paraesophageal LN )}

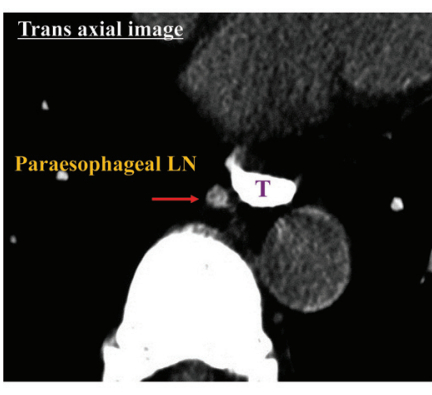

Mt 0-II c

pT1b (pSM3), pN1 (\# 108)

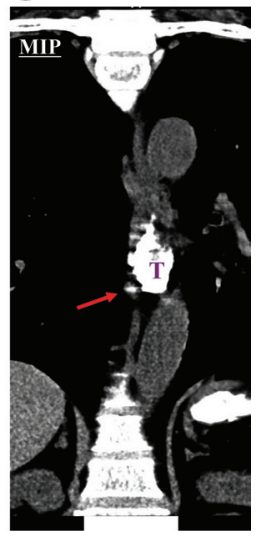

Fig. 6 Lymphatic routes and SLNs using CT lymphography.

The 3D images of CT lymphography (CTLG) showed that lymph flowed from the injection site into the SLNs. Multiple-angle views of the 3D CT image provided a comprehensive anatomy of these lymphatic pathways. Upward lymph flow and two right recurrent nerve nodes were detected clearly in the patient (A), In another case, one periesophageal node, which cannot be detected with lymphoscintigraphy because of shine-through phenomenon, was clearly visualized (B). Those SLNs were easily identified and removed under the guidance of 3D CT-LG images in all patients during surgery. The tumor occupied lymph node metastasis was translated in a mottled stain pattern, unstained swollen, or stain defect with rerouting lymph routes from CTLG images.
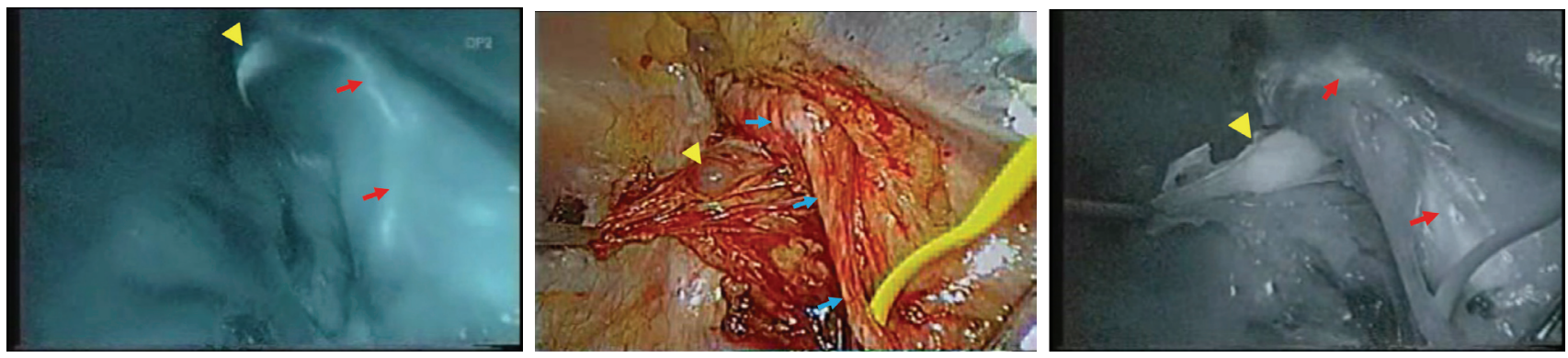

Fig. 7 Fluorescent navigation with ICG (indocianine green).

ICG (indocianine green) was injected into the submucosal layer beside the tumor, then fluorescent lymph flow (red arrow) and SLN (right recurrent nerve node: yellow arrow head) were clearly visualized and retrieved using an Infrared Endoscopic Camera System (OLIMPUS, Tokyo, Japan) and precise SLNs were sampled. ${ }^{52)}$

A: Intraoperative ICG fluorescent images clearly showed fluorescent lymph flow (red arrow) and SLN (right recurrent nerve node, yellow arrow head).

B: SLN (right recurrent nerve node, yellow arrow head) was retrieves along to the vagal nerve and right recurrent nerve (blue arrows) with referring CTLG image.

C: The SLN (right recurrent nerve node, yellow arrow head) and lymph flow (red arrow) was recognized with fluorescent through the camera.

superficial esophageal cancer. ${ }^{50-52)}$ The multiple-angle views of 3D, CT image sets provided a comprehensive anatomy of these lymphatic pathways (Fig. 6A and 6B). Our recent study about preoperative CTLG and intraoperative fluorescent navigation with ICG (indocyanine green) with Infrared Endoscopic Camera System (OLIMPUS, Tokyo, Japan) completed the precise detection of SLN with lymphatic routes from the tumor (Fig. 7A-7C). Tumor-occupied metastases were translated in unstained swollen, mottled stain pattern, or stain defect with rerouting lymph routes from the CTLG images. ${ }^{52)}$
The aim of surgical treatment for cancer is complete resection of the tumor-infiltrated organ including the regional lymph nodes. Accurate detection of SLN can achieve a selection of a more sophisticated tailor made approach.

We developed mediastinoscope assisted transhiatal esophagectomy (MATHE) ${ }^{53)}$ Esophagectomy can be performed with this procedure under clear visualization of the mediastinum, and LN sampling is feasible. If the sampled $\mathrm{LN}$ proved to be metastasis, conversion to a transthoracic radical operation with $\mathrm{LN}$ dissection is very easy. ${ }^{54)}$

The patient can make an individualized choice from a 
broader spectrum of therapeutic options including EMR, laparoscopic, thoracoscopic, MATHE, modified radical surgery, and typical, radical surgery with lymph node dissections. Ultra-staging, by the detection of micrometastasis, and the choice of an adequate adjuvant therapy improve the postoperative quality of life and survival. However, these issues require further investigation.

\section{Conclusion}

FDG-PET and CT scan are the gold standard to detect distant metastases of ESSC, but the metastatic node is difficult to diagnose from its size and SUV. After diagnosis with high resolution NBI endoscopy, the neighboring node should be examined with UES. US is also available for screening the neck and abdomen. Exact staging will be determined by the fine needle aspiration biopsy (FNAB) under EUS and US. For superficial cancers, indications for EMR and ESD should be sensitive because of the difficulty in making the diagnosis of Tlb cancer.. A detailed examination for finding residual tumor in the dissected specimen will predict an appropriate additional therapy. A new strategy like SLN navigation, in which CTLG is used, will supply more information about lymphatic routes and metastatic nodes. SLN navigation with ESD or MATHE might become a new, less invasive strategy for superficial esophageal cancer.

\section{References}

1) Saito H, Sato T, Miyazaki M. Extramural lymphatic drainage from the thoracic esophagus based on minute cadaveric dissections: fundamentals for the sentinel node navigation surgery for the thoracic esophageal cancers. Surg Radiol Anat 2007; 29: 531-42.

2) Kato H, Tachimori Y, Watanabe H, et al. Superficial esophageal carcinoma. Surgical treatment and the results. Cancer 1990; 66: 2319-23.

3) Endo M, Yoshino K, Kawano T, et al. Clinicopathologic analysis of lymph node metastasis in surgically resected superficial cancer of the thoracic esophagus. Dis Esophagus 2000; 13: 125-9.

4) Yoshida M. Recent development of studies on superficial cancer in Japan. Esophagus 2007; 4: 91-2.

5) Kodama M, Kakegawa T. Treatment of superficial cancer of the esophagus: a summary of responses to a questionnaire on superficial cancer of the esophagus in Japan. Surgery 1998; 123: 432-9.

6) Akiyama H, Tsurumaru M, Udagawa H, et al. Radical lymph node dissection for cancer of the thoracic esophagus. Ann Surg 1994; 220: 364-72; discussion 372-3.

7) Altorki N. En-bloc esophagectomy--the three-field dissection. Surg Clin North Am 2005; 85: 611-9, xi.

8) The Japan Esophageal Society. Guidelines for diagnosis and treatment for esophageal cancer. The 2 nd Ed,
Revised Version. Tokyo: Kanahara, 2007.

9) Rice TM. Staging of esophageal cancer: TNM and beyond. Esophagus 2010; 7: 189-195.

10) Sobin L, Gospodarowicz M, Wittekind Ch, eds.; International Union Against Cancer (UICC) TNM Classification of Malignant Tumours, 7th Edition, Oesophagus including Oesophagogastric Junction, New York: Wiley 2009; pp 66-72.

11) The Japan Esophageal Society: Japanese Classification of Esophageal cancer (The 10th Edition, Revised Version). Tokyo: Kanahara, 2008.

12) Natsugoe $S$. From uniformed treatment to individualized treatment for superficial esophageal cancer--what is potentially a new approach? Ann Thorac Cardiovasc Surg 2010; 16: 1-7.

13) Muto M, Minashi K, Yano T, et al. Early detection of superficial squamous cell carcinoma in the head and neck region and esophagus by narrow band imaging: a multicenter randomized controlled trial. J Clin Oncol 2010; 28: 1566-72.

14) Shimizu M, Nagata K, Yamaguchi H, et al. Squamous intraepithelial neoplasia of the esophagus: past, present, and future. J Gastroenterol 2009; 44: 103-12.

15) Thosani N, Singh H, Kapadia A, et al. Diagnostic accuracy of EUS in differentiating mucosal versus submucosal invasion of superficial esophageal cancers: a systematic review and meta-analysis. Gastrointest Endosc 2012; 75: 242-53.

16) Hsu WH, Hsu PK, Wang SJ, et al. Positron emission tomography-computed tomography in predicting locoregional invasion in esophageal squamous cell carcinoma. Ann Thorac Surg 2009; 87: 1564-8.

17) Bruzzi JF, Munden RF, Truong MT, et al. PET/CT of esophageal cancer: its role in clinical management. Radiographics 2007; 27: 1635-52.

18) Okada M, Murakami T, Kumano S, et al. Integrated FDG-PET/CT compared with intravenous contrast-enhanced CT for evaluation of metastatic regional lymph nodes in patients with resectable early stage esophageal cancer. Ann Nucl Med 2009; 23: 73-80.

19) van Vliet EP, Heijenbrok-Kal MH, Hunink MG, et al. Staging investigations for oesophageal cancer: a metaanalysis. Br J Cancer 2008; 98: 547-57.

20) Choi J, Kim SG, Kim JS, et al. Comparison of endoscopic ultrasonography (EUS), positron emission tomography (PET), and computed tomography (CT) in the preoperative locoregional staging of resectable esophageal cancer. Surg Endosc 2010; 24: 1380-6.

21) Vazquez-Sequeiros E, Foruny-Olcina JR. Linear EUS: the clinical impact of $\mathrm{N}$ staging in esophageal carcinoma. Minerva Med 2007; 98: 313-9.

22) Omloo JM, van Heijl M, Smits NJ, et al. Additional value of external ultrasonography of the neck after CT and PET scanning in the preoperative assessment of patients with esophageal cancer. Dig Surg 2009; 26: 43-9.

23) Hsu PK, Lin KH, Wang SJ, et al. Preoperative positron emission tomography/computed tomography predicts advanced lymph node metastasis in esophageal squamous cell carcinoma patients. World J Surg 2011; 35: 1321-6.

24) Shie P, Cardarelli R, Brandon D, et al. Meta-analysis: comparison of F-18 Fluorodeoxyglucose-positron emission tomography and bone scintigraphy in the 
detection of bone metastases in patients with breast cancer. Clin Nucl Med 2008; 32: 97-101.

25) Kato K, Muro K, Minashi K, et al. Phase II study of chemoradiotherapy with 5-fluorouracil and cisplatin for Stage II-III esophageal squamous cell carcinoma: JCOG trial (JCOG 9906). Int J Radiat Oncol Biol Phys 2011; 81: 684-90.

26) Udagawa H. Chemoradiotherapy: its effectiveness, toxicity, and perspective in the treatment of esophageal cancer. Ann Thorac Cardiovasc Surg 2009; 15: 359-61.

27) Ando N, Iizuka T, Ide H, et al. Surgery plus chemotherapy compared with surgery alone for localized squamous cell carcinoma of the thoracic esophagus: A Japan Clinical Oncology Group Study -JCOG 9204. J Clin Oncol 2003; 21: 4592-6.

28) Ando N, Kato H, Shinoda M, et al. A randomized trial comparing postoperative adjuvant chemotherapy with cisplatin and 5-fluorouracil versus preoperative chemotherapy for localized advanced squamous cell carcinoma of the thoracic esophagus (JCOG 9907). Ann Surg Oncol 2012; 19: 68-74.

29) Hirao M, Ando N, Tsujinaka T, et al. Influence of preoperative chemotherapy for advanced thoracic oesophageal squamous cell carcinoma on perioperative complications. Br J Surg 2011; 98: 1735-41.

30) Yoshida T, Seike J, Miyoshi T, et al. Preoperative chemotherapy with weekly docetaxel plus low dose cisplatin and 5-fluorouracil for stage II/III squamous cell carcinoma of the esophagus. Esophagus 2011; 7: 95-100.

31) Yamamoto Y, Yamai H, Seike J, et al. Prognosis of esophageal squamous cell carcinoma in patients positive for human epidermal growth factor receptor family can be improved by initial chemotherapy with docetaxel, fluorouracil, and cisplatin. Ann Surg Oncol 2012; 19: 757-65.

32) Kaur A, Dasanu CA. Targeting the HER2 pathway for the therapy of lower esophageal and gastric adenocarcinoma. Expert Opin Pharmacother 2011; 12: 2493-503.

33) Makino T, Doki Y, Miyata H, et al. Use of (18)F-fluorodeoxyglucose-positron emission tomography to evaluate responses to neo-adjuvant chemotherapy for primary tumor and lymph node metastasis in esophageal squamous cell carcinoma. Surgery 2008; 144: 793-802.

34) Wieder HA, Brücher BL, Zimmermann F, et al. Time course of tumor metabolic activity during chemoradiotherapy of esophageal squamous cell carcinoma and response to treatment. J Clin Oncol 2004; 22: 900-8.

35) Sandha GS, Severin D, Postema E, et al. Is positron emission tomography useful in locoregional staging of esophageal cancer? Results of a multidisciplinary initiative comparing CT, positron emission tomography, and EUS. Gastrointest Endosc 2008; 67: 402-9.

36) Wong R, Walker-Dilks C, Raifu A. Evidence-based guideline recommendations on the use of positron emission tomography imaging in oesophageal cancer. Clin Oncol (R Coll Radiol) 2012; 24: 86-104.

37) Misra S, Choi M, Livingstone AS, et al. The role of endoscopic ultrasound in assessing tumor response and staging after neoadjuvant chemotherapy for esophageal cancer. Surg Endosc 2012; 26: 518-22.

38) Jamil LH, Gill KR, Wallace MB. Staging and restaging of advanced esophageal cancer. Curr Opin Gastroenterol 2008; 24: 530-4.
39) Xu W, Shi J, Zeng X, et al. EUS elastography for the differentiation of benign and malignant lymph nodes: a meta-analysis. Gastrointest Endosc 2011; 74: 1001-9; quiz 1115.e1-4.

40) Kato H, Sato A, Fukuda H, et al. A phase II trial of chemoradiotherapy for stage I esophageal squamous cell carcinoma: Japan Clinical Oncology Group Study (JCOG9708). Jpn J Clin Oncol 2009; 39: 638-43.

41) Shitara K, Muro K. Chemoradiotherapy for treatment of esophageal cancer in Japan: current status and perspectives. Gastrointest Cancer Res 2009; 3: 66-72.

42) Kurokawa Y, Muto M, Minashi K, et al. Gastrointestinal Oncology Study Group of Japan Clinical Oncology Group (JCOG). A phase II trial of combined treatment of endoscopic mucosal resection and chemoradiotherapy for clinical stage I esophageal carcinoma: Japan Clinical Oncology Group Study JCOG0508. Jpn J Clin Oncol 2009; 39: 686-9.

43) Veronesi U, Paganelli G, Galimberti V, et al. Sentinelnode biopsy to avoid axillary dissection in breast cancer with clinically negative lymph-nodes. Lancet 1997; 349: 1864-7.

44)Langer I, Guller U, Hsu-Schmitz SF, et al. Sentinel lymph node biopsy is associated with improved survival compared to level I \& II axillary lymph node dissection in node negative breast cancer patients. Eur J Surg Oncol 2009; 35: 805-13.

45) Giuliano AE, Hunt KK, Ballman KV, et al. Axillary dissection vs no axillary dissection in women with invasive breast cancer and sentinel node metastasis: a randomized clinical trial. JAMA 2011; 305: 569-75.

46) Takeuchi H, Kitagawa Y. Sentinel node navigation surgery in upper gastrointestinal cancer: what can it teach us? Ann Surg Oncol 2011; 18: 1812-3.

47) Uenosono Y, Arigami T, Yanagita S, et al. Sentinel node navigation surgery is acceptable for clinical T1 and N0 esophageal cancer. Ann Surg Oncol 2011; 18: 2003-9.

48) Tangoku A, Yamamoto S, Suga K, et al. Sentinel lymph node biopsy using computed tomography-lymphography in patients with breast cancer. Surgery 2004; 135: 258-65.

49) Tangoku A, Seike J, Nakano K, et al. Current status of sentinel lymph node navigation surgery in breast and gastrointestinal tract. J Med Invest 2007; 54: 1-18.

50) Suga K, Shimizu K, Kawakami Y, et al. Lymphatic drainage from esophagogastric tract: feasibility of endoscopic CT lymphography for direct visualization of pathways. Radiology 2005; 237: 952-60.

51) Hayashi H, Tangoku A, Suga K, et al. CT lymphographynavigated sentinel lymph node biopsy in patients with superficial esophageal cancer. Surgery 2006; 139: 224-35.

52) Yuasa Y, Seike J, Yoshida T, et al. Sentinel lymph node biopsy using intraoperative indocyanine green fluorescence imaging navigated with preoperative CT lymphography for superficial esophageal cancer. Ann Surg Oncol 2012; 19: 486-93.

53) Tangoku A, Yoshino S, Abe T, et al. Mediastinoscopeassisted transhiatal esophagectomy for esophageal cancer. Surg Endosc 2004; 18: 383-9.

54) Tangoku A, Hayashi H, Kanamura S, et al. Lymph node metastases identified with mediastinoscopy in patient with superficial carcinoma of the esophagus. Surg Endosc 2000; 14: 595. 